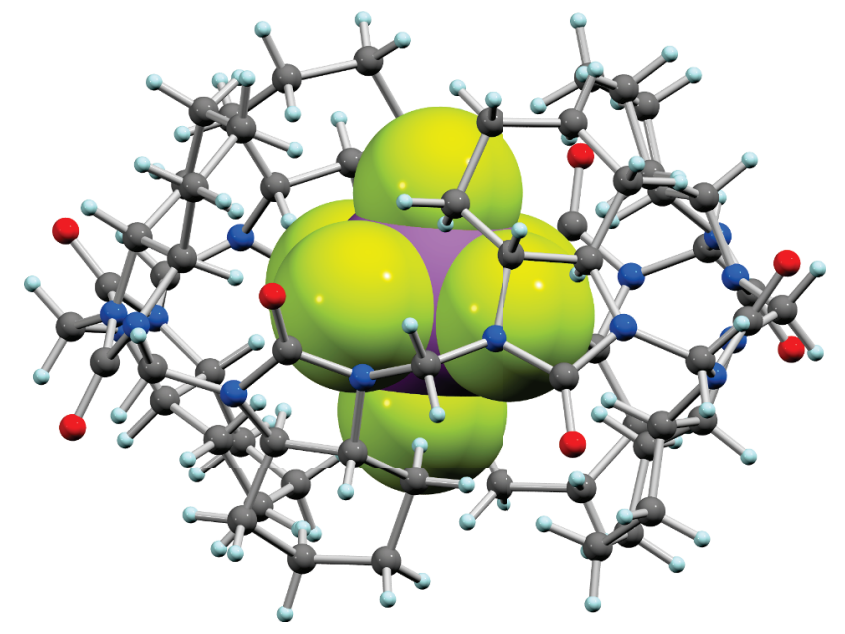

Figure 1. The structure of an inclusion complex of cycHC[8]: $\mathrm{SbF}_{6}^{-}$from X-ray diffraction analysis

Keywords: hemicucurbiturils, host-guest chemistry, anion binding

\section{MS36-P17 Shortest intermolecular contacts in crystals of organic compounds}

Michał Kaźmierczak ${ }^{1}$, Andrzej Katrusiak ${ }^{1}$

1. Department of Materials Chemistry, Faculty of Chemistry, Adam Mickiewicz University in Poznań. Umultowska 89b, 61-614 Poznań, Poland

email: kax@amu.edu.pl

The intermolecular interactions are one of the most important factor governing the formation of crystal structures.[i],[ii] Therefore characterization of the shortest contacts is extremely important for understanding this process, developing methods of crystal engineering[iii] and predicting the crystal structures.[iv] The investigation of the shortest contacts population reveals bimodal distribution and a clear distinction between strong and weak interactions. Further deconvolution, show that populations are sum of Gaussian-like distributions for specific intermolecular contacts (Fig. 1).[v] Fitting to correct function gave parameters, like mean distance and standard deviation, very useful for validating crystal structures. Moreover, we have found group of crystal structures which does not have any van der Waals contacts,[vi] according to Bondi.[vii] The existence of such population is an important input in discussion of van der Waals radii values, and can be starting point for their recalculation.

[i] Desiraju, G. R. Crystal Engineering: The Design of Organic Solids; Elsevier: Amsterdam, 1989.

[ii] Desiraju, G. R. A Bond by Any Other Name Angew. Chem., Int. Ed. 2011, 50, 52-59.

[iii] Nalini, V.; Desiraju, G. R. The Role of Non-bonded Interactions Involving Sulphur in the Crystal Engineering of $4 \AA$ Short Axis Structures. Unusual Topochemical Reactivity of 4-(4-chlorophenyl)-thiazole-2(1H)-thione. J. Chem. Soc., Chem. Commun. 1986, 1030-1032.

[iv] Price, S. L. Computed Crystal Energy Landscapes for Understanding and Predicting Or ganic Crystal Structures and Polymorphism. Acc. Chem. Res. 2009 , 42, 117-126.

[v] Kaźmierczak, $\mathrm{M}$ and Katrusiak, A. Bimodal Distribution of the Shortest Intermolecular Contacts in Crystals of Organic Compounds Crystal Growth \& Design 201414 (5), 2223-2229.

[vi] Kaźmierczak, M.; Katrusiak, A. The Most Loose Crystals of Organic Compounds J. Phys. Chem. C 2013, 117, 1441-1446.

[vii] Bondi, A. van der Waals Volumes and Radii. J. Phys. Chem. 1964, 68, 441-451.

[vii] Allen, F. H. The Cambridge Structural Database: A Quarter of a Million Crystal Structures and Rising. Acta Crystallogr., Sect. B: Struct. Sci. 2002,58 , 380-388. 


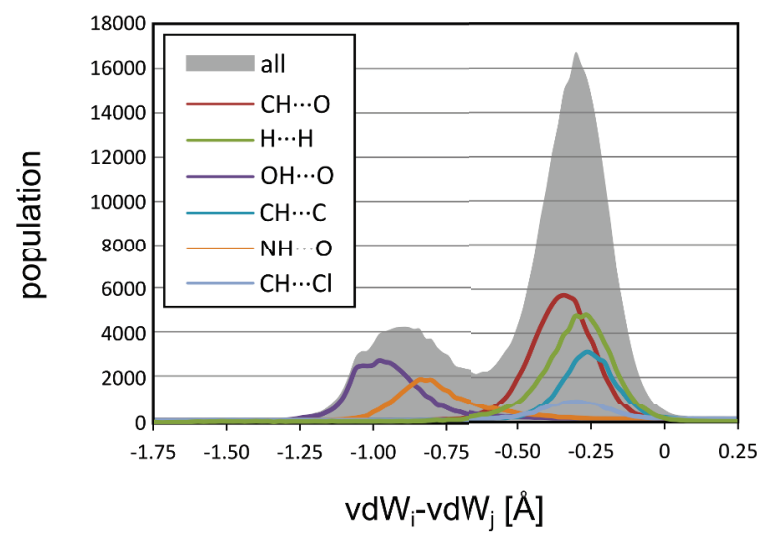

Figure 1. Distribution of six most popular types of shortest contacts in the CSD[viii] in function of small distances between van der Waals spheres.

Keywords: Intermolecular Interactions, Crystal Engineering

\section{MS36-P18 [ $\left.\mathrm{C}_{10} \mathrm{mim}\right][\mathrm{Cl}]$ : An ionic liquid, a liquid crystal and a crystallisation solvent}

Sofiane Saouane ${ }^{1}$, Francesca P. A. Fabbiani ${ }^{1}$

1. GZG, Abteilung Kristallographie, Georg-August-Universität Göttingen, Göttingen, Germany

email: ssaouan@gwdg.de

Ionic liquids (ILs) are molten salts below the boiling point of water. ${ }^{1}$ ILs are referred to as "designer solvents": ${ }^{2}$ they can be pre-tuned to desired physical and chemical properties through the numerous combinations of cations and anions. The sphere of interest in ILs has increased in the last decades. ILs' versatile properties enable their application in several fields, among them the pharmaceutical research where ILs are starting to attract interest as crystallisation solvents.

In a series of investigations of imidazolium-based ILs, ${ }^{4}$ the solid state behaviour of 1-decyl-3-methylimidazolium chloride, $\left[\mathrm{C}_{10} \operatorname{mim}\right][\mathrm{Cl}]$, has been studied using crystallisation and diffraction techniques at non-ambient conditions. The characterisation of the rich, hitherto unknown solid-state behaviour of $\left[\mathrm{C}_{10} \mathrm{mim}\right][\mathrm{Cl}]$ hydrates and liquid crystal phases are presented herein, along with preliminary results of adopting ILs as high-pressure crystallisation media for investigating drug polymorphism.

Keywords: ionic liquids, intermolecular interactions, high pressure, low temperature, crystallisation, hydrates. 